\title{
THEORETICAL LIGHT CURVES FOR TYPE IA SUPERNOVAE AND DETERMINATION OF THE HUBBLE CONSTANT
}

\author{
KOICHI IWAMOTO AND KEN'ICHI NOMOTO \\ Department of Astronomy. University of Tokyo \\ Yayoi 2-11-16. Bunkyo ward. Tokyo 113, JAPAN
}

The large luminosity $\left(M_{V} \approx-19 \sim-20\right)$ and the homogeneity in light curves and spectra of Type Ia supernovae(SNe Ia) have led to their use as distance indicators ultimately to determine the Hubble constant $\left(H_{0}\right)$. However, an increasing number of the observed samples from intermediateand high- $z(z \sim 0.1-1)$ SN Ia survey projects(Hamuy et al. 1996, Perlmutter et al. 1997) have shown that there is a significant dispersion in the maximum brightness ( $\sim 0.4 \mathrm{mag})$ and the brighter-slower correlation between the brightness and the postmaximum decline rate, which was first pointed out by Phillips(1993). By taking the correlation into account, Hamuy et al.(1996) gave an estimate of $H_{0}$ within the error bars half as much as previous ones.

To clarify the possible reason of this new observational fact, we calculated monochromatic light curves for a variety of explosion models of $\mathrm{SNe}$ Ia with a time-dependent, multi-frequency, and multi-angle radiative transfer code. The models applied here include a standard deflagration model W7 and a series of delayed detonation models with a parameterized transition density from deflagration to detonation. We find that amongst the Chandrasekhar mass white dwarf models the difference in the ${ }^{56} \mathrm{Ni}$ mass in delayed detonation models may reproduce the observed correlation due to the difference in thermal structure of the ejecta and the temperature dependence of opacities. As a result of light curve fit for some of the above samples of SNe Ia, we found the value of the Hubble constant to be $H_{0}=66 \pm 12$ $\mathrm{km} \mathrm{sec}^{-1} \mathrm{Mpc}^{-1}$ (Iwamoto 1997, Iwamoto et al. 1998)

\section{References}

Hamuy M. et al., 1996, AJ 112, 2398.

Iwamoto K., 1997, $\mathrm{PhD}$ thesis, University of Tokyo.

Iwamoto K. et al., 1998, in preparation

Perlmutter S. et al., 1997, ApJ 483, 565.

Phillips M.M. 1993, ApJ 413, L105. 